following morning) may allow the service to be "put to bed" by midnight, giving juniors at least some chance of getting some sleep.

AMALGAMATION OF UNITS

Small units (less than four consultants) may experience severe problems in reducing junior doctors' hours (for all but the first stage). The alternatives to closure are to have consultants working antisocial hours by being first on call or to share on call with other units (same specialty in other hospitals or other specialties in the same hospital).

DEFAULT LEAVE PERIODS

Some of the greatest problems are created when more than one junior wishes to take leave at the same time, especially at the end of the job. Leave periods must be written into the job contract signed at the time that the job is accepted. If junior doctors wish to change their leave the onus lies with them to prevent disruption of the service.

\section{Conclusion}

The box gives a logical plan for reducing junior doctors' hours. The changes in hospital service required to bring their hours back to realistic levels will need to be radical. However, they are long overdue and provide a tremendous opportunity to improve the service far beyond the issue of junior doctors' hours.

1 NHS Management Executive. Funior doctors. The new deal. London: NHS Management Executive, 1991.

University of London. Inappropriate duties for preregistration house officers. BM7 1991;302:facing p 570 (clinical research edition).

(Accepted 30 September 1992)

\title{
Quality of life measures in health care. III: resource allocation
}

\author{
D J Spiegelhalter, S M Gore, R Fitzpatrick, A E Fletcher, D R Jones, D R Cox
}

MRC Biostatistics Unit, Institute of Public Health, Cambridge CB2 2SR DJ Spiegelhalter, senior statistician

SM Gore, senior statistician

Department of Public Health and Primary Care, Oxford OX1 1NF

R Fitzpatrick, university

lecturer in medical sociology

Royal Postgraduate Medical School, London W12 0HS

A E Fletcher, senior lecturer in epidemiology

\section{Department of} Epidemiology and Public Health, University of Leicester, Leicester

D R Jones, professor of medical statistics

Nuffield College, Oxford OX1 1NF

D R Cox, warden

Correspondence to:

Dr Spiegelhalter.

BMF 1992;305:1205-9
In our two previous papers we discussed the use of measures of health related quality of life in clinical trials and in describing the health of populations. ${ }^{2}$ We now turn to the difficult issue of using quality of life measures for allocating limited resources among competing health care programmes. We concentrate on the quality adjusted life year (QALYs) since this approach has received most attention. QALYs use an index combining changes in survival and quality of life of patients (and possibly others) to assess benefit brought about by the funded programme. For each programme this benefit can be divided by its economic cost and the resulting ratio used to help allocate resources. QALYs can be used to choose between alternative programmes for treating the same patients or, more controversially, to choose among programmes targeted at different groups.

The underlying philosophy is that rationing of resources is inevitable and so it is best that it is explicit and accountable. ${ }^{3}$ To ration effectively some measures of output from health care must be established. However, it is important to distinguish between such general issues, with which most may agree, and the actual techniques used in published studies, which have been greatly criticised. For example, Williams describes a method to assess current life expectancy and quality of life in which quality of life is assigned a value " $q$ " on a 0 to 1 scale, 1 representing perfect health and 0 representing death. ${ }^{+}$Each future year is then counted as worth $q$, with the possibility of discounting the value of future years in a similar manner to that used for future costs. The total QALYs for the group are estimated with and without the intervention, and the difference between the two estimates is a single composite measure of the marginal output of the programme.

Williams emphasised the possible role of QALY analysis in the marginal allocation of additional resources, and North Western Regional Health Authority made an early unsuccessful attempt at allocating its discretionary specialties revenue reserve fund to its district health authorities. ${ }^{5}$ However, district health authorities are now considering such schemes ${ }^{6}$ at a more basic level, and in at least one case serious attempts are being made at implementation. ${ }^{7}$ In this article we focus on British applications, although the most extreme example of a QALY type analysis is that attempted in Oregon. ${ }^{8}$ Here 714 condition treatment pairs were placed in rank order and Oregon proposed to fund them from the Medicaid budget according to those ranks; the future of this project is now, however, in doubt.

The QALY analyses arouse strong opinions. Critics have questioned the assumptions underlying the procedure, doubted the quality of the data for calculations, raised ethical objections concerning equity, and questioned whose values were relevant and whether it is reasonable to compare different numbers of different groups of people by a single index..$^{9-14}$ Supporters of the QALY approach have said that current examples of QALY analysis are not definitive, that the method is still in development, and that it is intended only as an aid to decision making rather than a strict recipe. ${ }^{15-17}$ The lack of an alternative procedure has also been emphasised. ${ }^{16}$

To clarify the arguments below we identify the stages necessary to introduce a QALY system for resource allocation. We discuss whether progress from stage to stage is technically feasible or ethically desirable, and conclude with some recommendations for the appropriate future role of QALY type analyses.

\section{Adopting a formal approach to resource allocation}

Allocating scarce resources among competing health care programmes requires judgment about best use. The box gives the stages between extreme positions of completely informal judgment and rigid adherence to a formula.

Few would propose intuition unaided by explicit information concerning costs and benefits as an ideal method of allocating resources but it describes much of current practice (stage 1). Changes in the NHS are forcing decision making to progress to consider cost and health benefits, which in turn is highlighting the lack of suitable information (stage 2). The issue arises of whether it is solely the patients' outcomes that are relevant: for children, parents of young children, and handicapped and elderly people, the impact on the family and carers may need to be considered. Most people would agree that considering data on cost and health benefits is desirable, and most would wish to progress to collecting validated data (stage 3 ).

At stage 3 we would expect to know how many 


\section{Stages in developing QALY analysis}

1. Unaided intuitive judgment

2. Consideration of available data on costs and health benefits of each programme

3. Collection of validated costing, quality of life, and survival data for each programme

4. Use of common multidimensional quality of life measures across programmes

5. Collapse of quality of life measures to a single 0-1 scale

6. Summary of survival benefits as single measure

7. Combination of quality of life and survival data into single measure-for example, the quality adjusted life year (QALY)

8. Decisions made by ranking programmes according to their return in QALYs per pound and funding strictly according to rank order.

Stage 6 does not concern quality of life and so could be placed before stage 4 .

patients could potentially be treated in each programme, their initial health status, and the distribution of outcomes. This multidimensional information would then be summarised and compared among specialties. Quality of life should be measured with disease specific instruments ${ }^{12}$ and described with familiar outcome measures. Although good information could eventually be obtained for costs, it is more difficult for outcome measures. Often the information is simply not available-for example, the long term effects of heart transplantation in children. If we wish to generalise the approach outside treatment programmes it is unclear how to measure the health benefits of providing laboratory services, a computer information system, or even redecoration. A regional health authority found that it was not feasible to attempt to assess outcome in almost half their competing bids, ${ }^{5}$ and Coast found similar problems when trying to use published results as a basis for QALY calculations. $^{18}$

Nevertheless, the need for validated information fits closely with the current focus on continuous audit: if successful bids are accompanied by a claimed benefit the accuracy of the claim should be checked. This follows the Deming philosophy of everyone in an organisation monitoring quality. ${ }^{19}$ It does not mean that all patients must be followed up in detail-sample survey techniques should be much more widely used.

To compare results across programmes requires common measures for quality of life that can be used across all specialties (stage 4 ). The most popular in Britain is the two dimensional "disability-distress" scale developed by Rosser and Kind. ${ }^{20}$ Recently "physical discomfort" has been added as a third dimension, and Rosser et al have further subdivided these dimensions into 44 scales giving 107 descriptors of states. ${ }^{21}$ This index of health related quality of life (IHQL) therefore provides a detailed hierarchical description of health.

At the other extreme, others have developed a simple "Euroqol" instrument that has six dimensions (for example, mobility and self-care) each with only two or three levels. This has been tried out in several European communities, ${ }^{16}$ although neither the IHQL nor the Euroqol has been validated according to standard criteria. ${ }^{\prime}$ There is clearly a difficult balance between a comprehensive and lengthy instrument that tries to be sensitive within all clinical areas, and a simple technique that may be considered too crude within specialties: for example, the Euroqol mood dimension is a yes or no response to the question,
"Anxious or depressed?" which seems inadequate for evaluating psychiatric services.

\section{Summary measures}

To collapse quality of life scores into a single measure each state of health described by a global instrument must be assigned a number between 0 and 1 (stage 5). Many techniques exist for doing this, such as category rating, magnitude estimation, time trade off, and standard gamble. ${ }^{22}$ Considerable variability between techniques and between individuals has been identified. For example, Williams ${ }^{4}$ and many others used the initial values for the Rosser index derived from 70 people, 211 but recently values obtained from nearly 300 members of the general public by different techniques have been reported. ${ }^{16}$ These two sets are surprisingly different-for example, the value assigned to "moderate distress and unable to work' changed from $0.9^{20}$ to $0 \cdot 35 .{ }^{16}$ Nevertheless, if states rated zero are ignored then the two sets of values show a high correlation, which suggests that if mortality was not included they would give similar rankings.

This supports the argument of Carr-Hill that including death on a scale forces choices that cannot be realistically considered by subjects, making them reluctant to answer and their responses rather arbitrary. ${ }^{93}$ For Euroqol subjects were asked to mark various states of health on a "thermometer" between best possible (100) and worst imaginable (0) states of health. Among the $21 \%$ providing usable responses in a Swedish study, ${ }^{2+}$ the median score for death was 0 , while being anxious or depressed was placed at a median of 75 . The consequences of such an assessment will be explored later.

In contrast, Rosser et al use a hierarchical approach which largely avoids direct comparisons with death. ${ }^{21}$ From this can be derived a "disutility" specific to any positive response in the questionnaire, each being subtracted from 1 to obtain a final global measure of quality of life. However, the model contains strong assumptions $^{925}$ which can lead to implausible weights

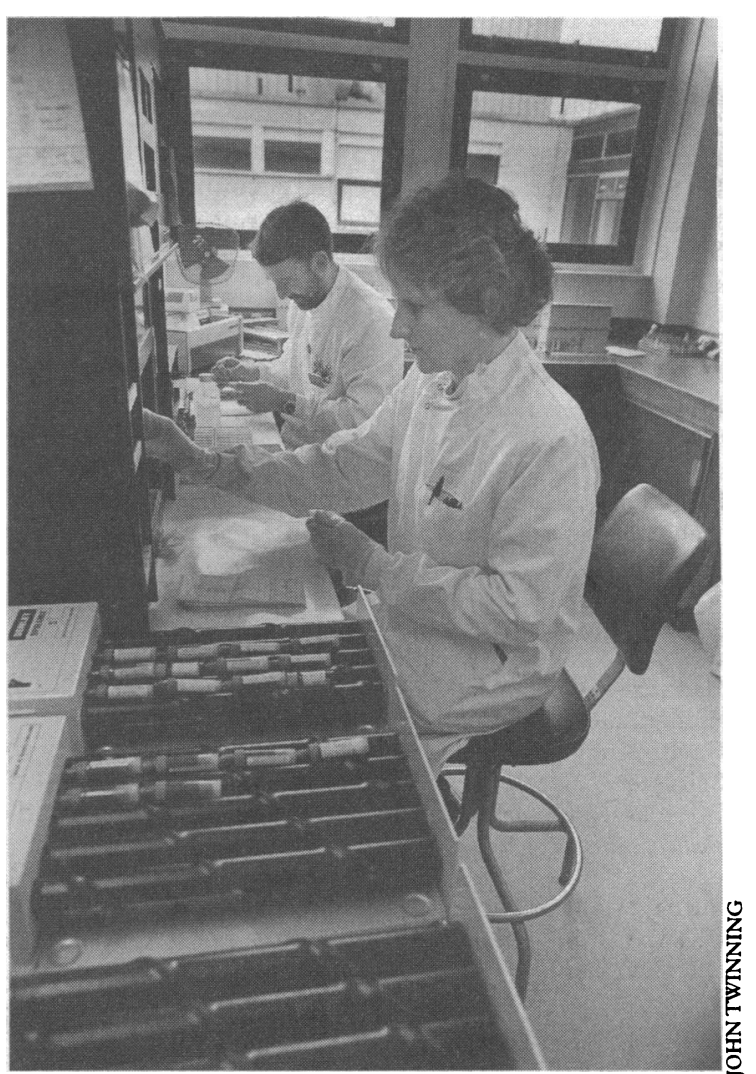

It's difficulty to apply quality of life measures to laboratory and other services 
-for example, in a reported sample, feeling a lack of ambition was assigned $45 \%$ more disutility than complete bowel incontinence. ${ }^{21}$

Both the Rosser index and Euroqol rely on ratings provided by the general public. Such population values may not reflect those of patients in general, let alone those of the patient whose treatment will be influenced. " In particular, healthy younger people may have an inappropriate view of what is important to elderly people. ${ }^{26}$ Although various surveys may be used for information, the decision making body must decide on the appropriateness of the values used and the sensitivity of the conclusions.

\section{Survival analysis}

Since length of survival cannot be predicted with certainty, any measure of survival benefit is essentially an average of survival distributions (stage 6). The steps within the standard QALY analysis are (a) estimate the survival distribution for patients with and without intervention, $(b)$ value each future year according to a fixed discount rate, $(c)$ calculate change in discounted life expectancy, $(d)$ add up over the group.

We make the major assumption that if validated data on quality of life and survival are collected the first step can be achieved. The second step reflects the perception that years in the distant future are not worth as much as the immediate future, although a suitable discount rate is the subject of considerable controversy (box).

Figure 2 shows the discounted life expectancy for average women of different ages derived from life tables for England and Wales. ${ }^{31}$ Clearly older people do not have the capacity for benefit ${ }^{11}$ of younger people, in that they can hope to achieve only a limited number of QALYs no matter how effective the intervention is; this has led to the accusation that the QALYs are agist. ${ }^{1+}$ While a low or zero discount rate will favour services aimed at the young, figure 2 shows that this differential will be almost eliminated by substantial discounting.

Any summary measure of a survival distribution, of which discounted life expectancy is only one choice, reflects a value judgment. ${ }^{16} \mathrm{~A}$ possible alternative is to express gain in life expectancy as a fraction of that for an average person of the same age. Thus an expected gain of 12 life years for a 20 year old would be equivalent to a gain of three life years for a 70 year old, since each is about $20 \%$ of the life expectancy. This measure also reflects the perception that a person's value of a year may depend strongly on context. ${ }^{32}$ Adoption of such a measure would tend to favour treatment for elderly people.

Simple addition of benefit in each person is in one sense egalitarian, in that everyone is weighted equally, and summarising by a cumulative output reflects the utilitarian philosophy underlying QALY analysis. Alternatively, a deontological approach emphasises the right of each person to care, and would not aggregate and rank groups according to their potential benefit. With this view everyone is given good (but not necessarily the greatest) treatment, thus saving lives rather than lifetimes. ${ }^{1+26}$ The standard QALY approach may be egalitarian, but it does not encourage equity, ${ }^{913}$ it is possible to stay within the QALY approach but invent a means of aggregation that reflects differing perspectives-for example, weighting individuals differently ${ }^{16}$-although this is likely to be particularly controversial.

\section{Problems of combining quality of life and survival}

Combining quality of life and survival scores to produce a single measure can lead to problems

\section{Discounting quality of life values}

In economic analyses costs are discounted to their net present value to reflect the fact that delays in expenditure reduce the real cost. For example, assuming a $6 \%$ interest rate, $£ 1$ now is of equal value to being guaranteed $£ 1.06$ after one year, $£ 1.06 \times 1.06$ in two years, and so on. Hence $£ 100$ committed in 10 years is equivalent to only $100 / 1 \cdot 06^{11}=£ 56$ that has to be spent now. Similar techniques can be used to discount the value of life years to be gained in the future. Figure 1 shows the discounted current value for future life years resulting from different discount rates. For example, with a $5 \%$ discount rate, preventive medicine that will extend a life of a 25 year old from 55 to 75 years will "buy" 20 additional years starting 30 years in the future. Figure 1 shows this has a net current value of only 3.1 years, equivalent to a gain of 3.5 years obtained immediately for an acutely ill patient. Substantial discounting therefore results in preference for immediate over delayed benefits.

The discount rate for health benefits is usually matched to that used for cost: a study of alternative strategies for lowering serum cholesterol concentration, future life years were discounted at $7 \%$ as "recommended by the Norwegian treasury." There has been recent controversy over this, ${ }^{2 *}$ with Department of Health economists suggesting a discount rate at or close to zero. ${ }^{29}$ They argue that there is no reason to relate health benefits with income, and a zero discount rate does not logically lead to eternally delayed investment. Their opinions have been disputed from an economic perspective. ${ }^{30}$

If we consider individual decision making, a plausible basis for discounting comes from the observation that generally people prefer to avoid risks of early death even if their life expectancy might be increased by taking that risk. For example, consider a 50 year old woman who has a life expectancy of only 13 years. An operation could give her normal life expectancy (age 80 ), but it has a $50 \%$ failure rate, and failure leads to a life expectancy of only two years. Without discounting, the operation is preferred since its expected value $(0.5 \times 30+0.5 \times 2=16)$ is greater than no operation. However, a $5 \%$ discount rate makes it preferable to forgo the operation, which would match many people's preferences. Attitudes to risk vary widely, ${ }^{\circ}$ and Rosser et al suggest there should be little discounting over the first 10 years. ${ }^{21}$ In addition, even if individuals do display substantial discounting in their own preferences, it does not necessarily follow that this should be directly reflected in an aggregate outcome measure for a community.

(stage 7). We will consider some clearly fictitious examples of potential programmes bidding for funding, simplified to allow the issues to be brought into focus.

1. To provide 100 middle aged women with short term treatment to relieve anxiety or depression (assigned $q=$ 0.75 ) estimated to remove symptoms for on average seven months.

2. To try out a new neurosurgical technique on 100 middle aged patients with the same symptoms as in (1). If successful it will relieve symptoms of life, but it carries a $24 \%$ risk of death during operation.

3.To provide 10 patients with advanced cancer with treatment that will give them two extra years of reasonable quality life $(q=0 \cdot 75)$.

4. To carry out one heart transplant on a 5 year old child, who without the operation is expected to live two years with poor quality $(q=0 \cdot 50)$; if the child survives the postoperative period (there is $20 \%$ initial mortality) he or she will return to full healthy life.

Assuming a $5 \%$ discount rate for future years, all four options will provide about 15 QALYs. Are these outputs equally desirable? Two concepts of equity 
seem to be at stake. Firstly, in options 1 and 2 the patients are similar to start with but while one option guarantees a small benefit for all, the second envisages large variation among patients. Uniformity of benefit also makes option 3 seem attractive. Option 2 also shows the problems in trading off mortality and quality of life: did those who rated anxiety or depression at $q=$ 0.75 realise that this could be used to justify such a high operative mortality? Similar examples have been discussed within the context of clinical trials. ${ }^{29}$

The second aspect of equity concerns the numbers and initial health of patients in each option: should resources be concentrated on the patients who can benefit most? A simplistic QALY analysis could obscure such issues rather than clarify them.

If we change the discount rate to $0 \%$, we obtain QALY benefits for options 1 to 4 of $15,30,15$, and 57 respectively, greatly favouring interventions with possible long term consequences. A discount rate of $10 \%$ gives scores of $15,10,15$, and 8 , thus representing strong aversion to risk of early death. While this example is clearly highly stylised, it shows that QALY analyses can be extremely sensitive to discount rate, and that suitable quality of life assessments for trade off of mortality and morbidity are difficult to obtain. Indeed, all such rankings will probably provoke disagreements, partly because a person's value structure is unlikely to obey the simplifying assumptions underlying the usual quality adjusted life year analysis. ${ }^{1221}$ More importantly in a QALY analysis quality of life values are aggregated over uncertainty, over time, and over groups of differing size to produce a single measure, and it is implausible that any values whatever the assessment procedure, can then give rise to appropriate consequences. ${ }^{33}$

Some have argued that many such objections could be avoided by making the procedure more elaborate. ${ }^{163435}$ For example, to deal with accusations that maximising QALYs leads to a Thatcherite preference for the fortunate, ${ }^{14}$ Wagstaff has suggested a non-linear "social welfare function" in which, for example, a gain from 1 to 2 QALYs is worth more than a gain from 10 to $11 .{ }^{36}$ Other equity considerations could be explicitly built into measures of output. But from the perspective of quality of life, there remains the crucial point that the means of assessing values from individuals does not reflect the use to which they will be put, and hence could give rise to the kind of dubious conclusions described above. If quality of life values are constructed rather than measured, then elicitation should be couched directly in terms of realistic, although necessarily simplified, resource allocation decisions.

The final stage in use of QALYs is strict adherence to league tables, requiring the decision making body to fund fully one programme before considering the next on the list, even if only a few people would certainly benefit (stage 8 ). Even health economists warn against such a rigid approach, ${ }^{35}{ }^{37}$ and most would see a QALY analysis as an aid in decision making that clarified the issues and led to more accountable, consistent decisions. ${ }^{5}$ Drummond emphasised that an essential aspect is a careful sensitivity analysis of the crucial numerical inputs, ${ }^{37}$ as shown in a study of the treatment of low birthweight babies. ${ }^{38}$

\section{Conclusions}

The poor state of information management within the NHS will make even validated data collection difficult. At the same time, it may be appropriate to explore QALY analyses when considering resource allocation within programmes-for example, in setting priorities within a waiting list for kidney transplants, in which there is a disease specific outcome measure, a homogeneous population, and groups of different sizes and composition are not being compared. The issues are then similar to those faced in a clinical trial, in which there are still problems in aggregating quality and length of life. ${ }^{32}$ In particular, we question the appropriateness of current techniques for exploring values to be placed on quality of life measures that include death and believe such valuations should be elicited within the context of resource allocation decisions.

Even if reasonable population valuations on quality of life can be obtained, formal comparison of different patient groups is fraught with difficulties. Dowie suggests first making a political decision on how much should be spent in different areas of health care, and using careful QALY type analyses within these areas ${ }^{3+}$; possible categories might be research and development, prevention, chronic care, and acute services. Not having to make explicit trade offs between such categories avoids many of the difficulties identified above, while still encouraging an accountable analysis within each area.

Buxton rightly states that any QALY analysis is inevitably a simplification, ${ }^{35}$ but the crucial issue is whether this will lead to unwise or unfair decisions. The possibility of misleading results arising from an apparently "scientific" procedure is not just a technical problem that can be solved by yet larger surveys but requires continual public scrutiny of what lies behind each step in the development of composite outcome measures. Any formal approach must clarify rather than obfuscate the issues, and this would seem to require that decision makers retain direct control over the aggregation of basic outcome data. While we strongly recommend that attempts are made to progress to higher stages of decision making, elaborate calculations are, in our view, not justified until much better data are available on outcome measures, and we suggest that much more effort should be expended on this stage.

1 Fitzpatrick R, Fletcher AE, Gore SM, Jones DR, Spiegelhalter DJ, Cox DR Quality of life measures in health care. 1. Applications and issues in measurement. BMF 1992;305:1074-7.

2 Fletcher A, Gore SM, Spiegelhalter DJ, Jones DR, Fitzpatrick R, Cox DR Quality of life measures in health care. 2. Design, analysis and interpretation. BM7 1992;305:1145-8

3 Smith R. Rationing: the search for sunlight. BMf 1991;303:1561-2.

4 Williams A. Economics of coronary artery bypass grafting. BM7 1985;291: $326-9$

5 Allen D, Lee RH, Lowson K. The use of QALYs in health service planning Intermational Yournal of Health Planning and Management 1989;4:261-73.

6 Dean M. Is your treatment economic, effective and efficient? Lancet 1991;337; 480-1.

7 Walker P. Will quality-adjusted life-years be useful to a purchaser of health services? In: Hopkins A, ed. Measures of the quality-of-life. London: Royal College of Physicians, 1992.

8 Dixon J, Welch HG. Priority setting: lessons from Oregon. Lancet 1991;337: 891-4

9 Carr-Hill RA. Assumptions of the QALY procedure. Soc Sci Med 1989;28 $469-77$.

10 Carr-Hill RA. Allocating resources to health care: is the QALY (quality adjusted life vear) a technical solution to a political problem? Int $f$ Health Serv 1991;21:351-63.

11 LaPuma J, Lawlor EF. Quality adjusted life year: ethical implications for physicians and policymakers. $\mathcal{F} A M A$ 1990;263:2917-22.

12 Loomes G, McKenzie L. The use of QALYs in health-care decision making. Soc Sci Med 1989;28:299-308.

13 Smith A. Qualms about QALYs. Lancet 1987;1:1134-6.

14 Harris J. Unprincipled QALYs. F Med Ethics 1991;17:185-8.

15 Williams A. Is the QALY a technical solution to a political problem? Of course not! Int 9 Health Serv 1991;21:365-9.

16 Williams A, Kind P. The present state of play about QALYS. In: Hopkins A ed. Measures of the quality-of-life. London: Royal College of Physicians, 1992:21-34

17 Cubbon I. The principle of QALY maximisation as the basis for allocating health care resources. F Med Ethics 1991;17:181-4.

18 Coast J. Reprocessing data to form QALYs. BMY 1992;305:87-90.

19 Berwick DM, Enthoven A, Bunker JP. Quality management in the NHS: the doctor's role-1. BMF 1992;304:235-9.

20 Rosser R, Kind P. A scale of valuations of states of illness: is there a social consensus? Int $\mathcal{F}$ Epidemiol 1978;7:347-58.

21 Rosser R, Cottee M, Rabin R, Selai C. Index of health-related quality-of-life In: Hopkins A, ed. Measures of the quality-of-life. London: Royal College of Physicians, 1992:81-9.

22 Torrance GW. Utility approach to measuring health-related quality-of-life. fChron Dis 1987;40:593-600.

23 Carr-Hill RA. A good measure for Eurohealth? Health Service fournal $1991 ; 101: 25-8$ 
24 Brook RG, Jendleg S, Lindgren B, Persson U, Bjork S. Euroqol: health related quality of life measurement. Results of the Swedish questionnaire exercise. Health Policy 1991;18:37-48.

25 Keeney RL, Raiffa H. Decisions with multiple objectives: preferences and value trade-offs. New York: Wiley, 1976.

26 Grimley-Evans J. Quality-of-life assessments and elderly people. In: Hopkins A, ed. Measures of the quality-of-life. London: Royal College of Physicians, A, ed. Measur

27 Kristiansen IS, Eggen AE, Thelle DS. Cost effectiveness of incremental programmes for lowering serum cholesterol concentration: is individual intervention worthwhile? BMf 1991;302:1119-22.

28 Discounting health care: only a matter of timing? Lancet 1992;340:148-9.

29 Parsonage M, Neuberger H. Discounting and health benefits. Health Economics 1992;1:71-6.

30 Cairns J. Discounting and health benefits: another perspective. Health Economics 1992;1:76-9.

31 Office of Population Censuses and Surveys. English life tables. No 14. London: HMSO, 1987.
32 Normand C. Economics, health, and the economics of health. BMf 1991; 303:1572-7.

3 Cox DR, Fitzpatrick R, Fletcher AE, Gore SM, Spiegelhalter DJ, Jones DR Quality-of-life assessment: can we keep it simple? fournal of the Royal Quatistical Society Series A 1992;155:353-93.

34 Dowie J. The ethics of allocating health resources. Sydney: New South Wales Health Department, 1991. (State Health Publication No (HPA)91-58.)

35 Buxton MJ. Are we satisfied with QALYs? In: Hopkins A, ed. Measures of the quality-of-life. London: Royal College of Physicians, 1992:41-9.

36 Wagstaff A. QALYs and the equity-efficiency trade-off. Journal of Health Economics 1991;5:1-30.

37 Drummond MF. Resource allocation decisions in health care: a role for quality of life assessments? F Chron Dis 1987;40:605-16.

38 Boyle MH, Torrance GW, Sinclair JC, Sargent PH. Economic evaluation of neonatal intensive care of very-low-birth-weight infants. $N$ Engl $f \mathrm{Med}$ 1983;308:1330-7.

(Accepted 9 September 1992)

\title{
Reforming the Department of Health's research and development policy: from the devil to the deep blue sea?
}

\author{
Alan Maynard, Trevor Sheldon
}

\begin{abstract}
Summary
Research into health and social services in Britain is largely funded by the Department of Health. Regional NHS research and development has recently been reformed and a new report now proposes replacement of the 13 research units funded by the department with three or four large multidisciplinary centres. Evidence to support such a step is lacking, and many criticisms of the existing units arise from poor departmental planning rather than deficiencies of the units themselves. Large units may make research less responsive to the department's needs, and it is essential that the proposed new structure is thoroughly evaluated before it is introduced.
\end{abstract}

Since the reform of the Department of Health's research and development division and the appointment of Professor Michael Peckham as its director, the profile of health and health care research has improved inside and outside the department. This development has been assisted by a demand for knowledge about "what works" in health care by purchasers and providers, who are required to deliver demonstrably cost effective care to patients in the newly reformed NHS.

The evolving reforms of the department's research and development policy, like the NHS reforms themselves, are bold, radical, and unproved. Careful consideration of their potential effectiveness is essential and it is important to proceed carefully and evaluate systematically.

The research and development policy announced in $1991^{1}$ created 14 regional bureaucracies to manage the existing and largely inadequate regional research functions. This structure is nearing completion. It is consuming considerable sums in management and may focus research activity and funding more effectively on NHS policy needs. The case for piloting and evaluating such a structure was, as with the case of the NHS reforms, rejected even though no evidence existed to support the structure and the merits of a unitary national institute of health, on the American model, were considerable and extensively explored in various forums. ${ }^{2}$

\section{Proposed abolition of research units}

Following the reform of regional NHS research and development it is proposed that the 13 research units funded by the Department of Health are abolished. ${ }^{34}$

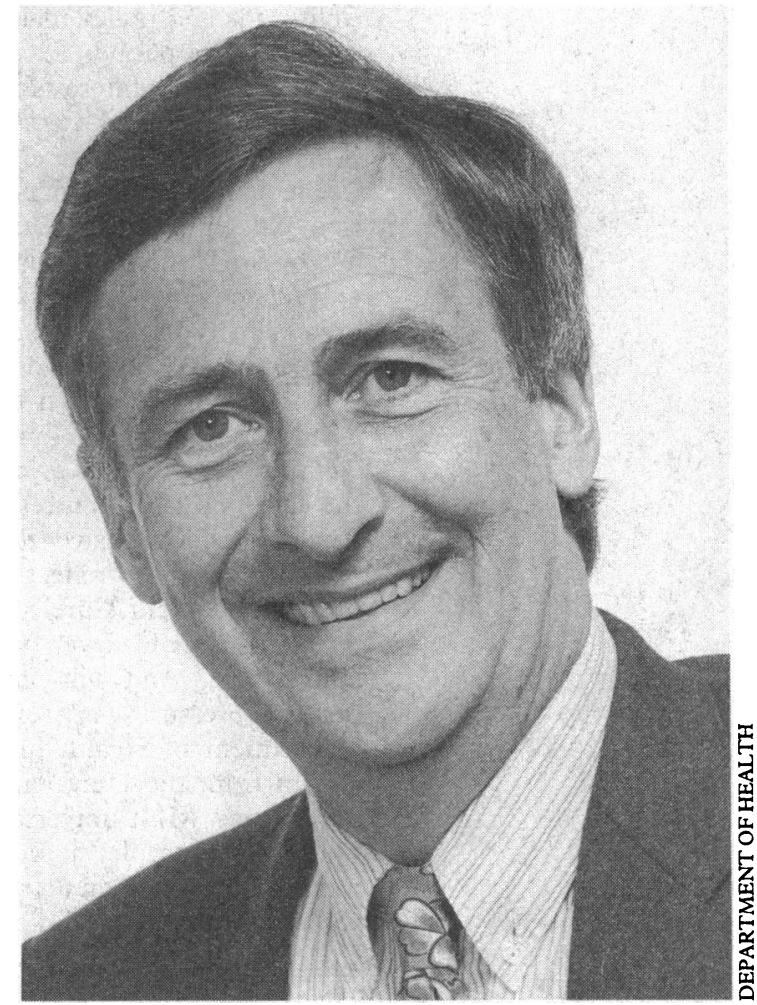

Professor Michael Peckham has raised the profile of health service research

The department commissions over $30 \%$ of noncommercially funded research into health and persona social services in England. The units currently receive f6m, one third of the research and development division's budget. They are as diverse as Oxford's National Perinatal Epidemiology Unit, the York Centre for Health Economics, the Thomas Coram research unit, and the Childhood Cancer Research Unit.

These units may be replaced by three to four multidisciplinary centres chosen by competitive bidding. As 10 of the 13 units currently receive over $60 \%$ of their funding from the Department of Health this will mean the closure or severe contraction of nationally and in some cases internationally renowned units which form an important element of the limited health and social service research infrastructure.

Why change? The authors of the report argue that the 13 units cover too wide a field, that there is a danger of isolation and a need for greater collaboration, that 